\title{
LETTER
}

Lymphoma

\section{STAT3 and TP53 mutations associate with poor prognosis in anaplastic large cell lymphoma}

\author{
Cosimo Lobello $\mathbb{D}^{1} \cdot$ Boris Tichy $^{1} \cdot$ Vojtech Bystry $^{1} \cdot$ Lenka Radova $^{1} \cdot$ Daniel Filip ${ }^{1,2} \cdot$ Marek Mraz $^{1,2}$. \\ Ivonne-Aidee Montes-Mojarro ${ }^{3}$. Nina Prokoph $\mathbb{D}^{4} \cdot$ Hugo Larose $\mathbb{D}^{4} \cdot$ Huan-Chang Liang $\mathbb{D}^{5} \cdot$ Geeta G. Sharma ${ }^{6}$. \\ Luca Mologni $^{6} \cdot$ David Belada $^{7} \cdot$ Katerina Kamaradova $^{8} \cdot$ Falko Fend $^{3}$. Carlo Gambacorti-Passerini $\mathbb{1}^{6}{ }^{6}$ \\ Olaf Merkel ${ }^{5} \cdot$ Suzanne D. Turner $\mathbb{1}^{1,4} \cdot$ Andrea Janikova $^{2} \cdot$ Sarka Pospisilova $\mathbb{C}^{1,2}$
}

Received: 7 April 2020 / Revised: 1 October 2020 / Accepted: 9 November 2020 / Published online: 27 November 2020

(c) The Author(s) 2020. This article is published with open access

\section{To the Editor:}

Systemic anaplastic large cell lymphoma (sALCL) encompasses two distinct clinical entities of T-cell non-Hodgkin lymphoma: anaplastic lymphoma kinase-positive (ALK+) ALCL and ALK-negative (ALK-) ALCL. These entities are characterized by either the presence or absence of an ALK translocation. It has been reported that ALK+ ALCL has a better prognosis compared to ALK-, with a 5-year

Supplementary information The online version of this article (https:// doi.org/10.1038/s41375-020-01093-1) contains supplementary material, which is available to authorized users.

Sarka Pospisilova

sarka.pospisilova@ceitec.muni.cz

1 Center of Molecular Medicine, Central European Institute of Technology (CEITEC), Masaryk University, Brno, Czech Republic

2 Department of Internal Medicine-Hematology and Oncology, University Hospital Brno and Medical Faculty MU, Brno, Czech Republic

3 Institute of Pathology and Neuropathology and Comprehensive Cancer Center Tübingen, Eberhard Karls University, Tübingen, Germany

4 Division of Cellular and Molecular Pathology, Department of Pathology, University of Cambridge, Cambridge, UK

5 Department of Pathology, Medical University of Vienna, Vienna, Austria

6 Department of Medicine and Surgery, University of MilanoBicocca, Monza, Italy

7 4th Department of Internal Medicine-Hematology, Charles University Hospital and Faculty of Medicine, Hradec Králové, Czech Republic

8 Fingerland Department of Pathology, Charles University Hospital and Faculty of Medicine, Hradec Králové, Czech Republic overall survival (OS) of 70-80\% versus $40-60 \%$, respectively, [1-3]. Furthermore, more than $30 \%$ of ALK + ALCL patients relapse $[4,5]$. Despite the distinction between the two sALCL subtypes, frontline treatment for adults is similar and is based on CHOP or CHOEP, instead pediatric ALCL patients are mainly treated following the ALCL99 protocol [6-8]. Whilst high-throughput genomic studies in sALCL have shown recurrent genetic alterations, their association with outcome has not been fully investigated [9-13].

In this study, the mutational landscape of sALCL patient tumors was investigated to discover potential biomarkers that may improve risk stratification and patient management.

A cohort of 82 sALCL patient tumors (47 ALK+ and 35 ALK-) and 6 ALCL cell lines (4 ALK+, 2 ALK-) (Table S1) were subjected to deep targeted next-generation sequencing analyzing the whole coding regions of 275 cancer related genes (Table S2). The average depth achieved across all the samples sequenced was $\sim 2000 x$. Sequencing data are available at Sequence Read Archive (https://www.ncbi.nlm.nih.gov/sra/, SRA identifier PRJNA602225).

Male subjects were predominant in both subgroups of our cohort, $57.4 \%$ in ALK + versus $67.6 \%$ in ALK-. ALK + patients were significantly younger than ALKpatients with an average age of 22.7 (3-61) and 55.2 (27-81) years, respectively. ALK + ALCL patients had a longer survival than ALK - ALCL with a 7-year OS of $77.6 \%$ and $46.7 \%$, respectively, and with 7-year progression free survival (PFS) being comparable at $58.7 \%$ for ALK+ and $44.1 \%$ for ALK - patients (Fig. S1). The first line of treatment for all the adult patients was systemic chemotherapy, and most of the childhood ALK + ALCL patients $(80 \%)$ were treated following the ALCL99 or ALCL98 protocols. Although ALK+ patients have a longer 
A

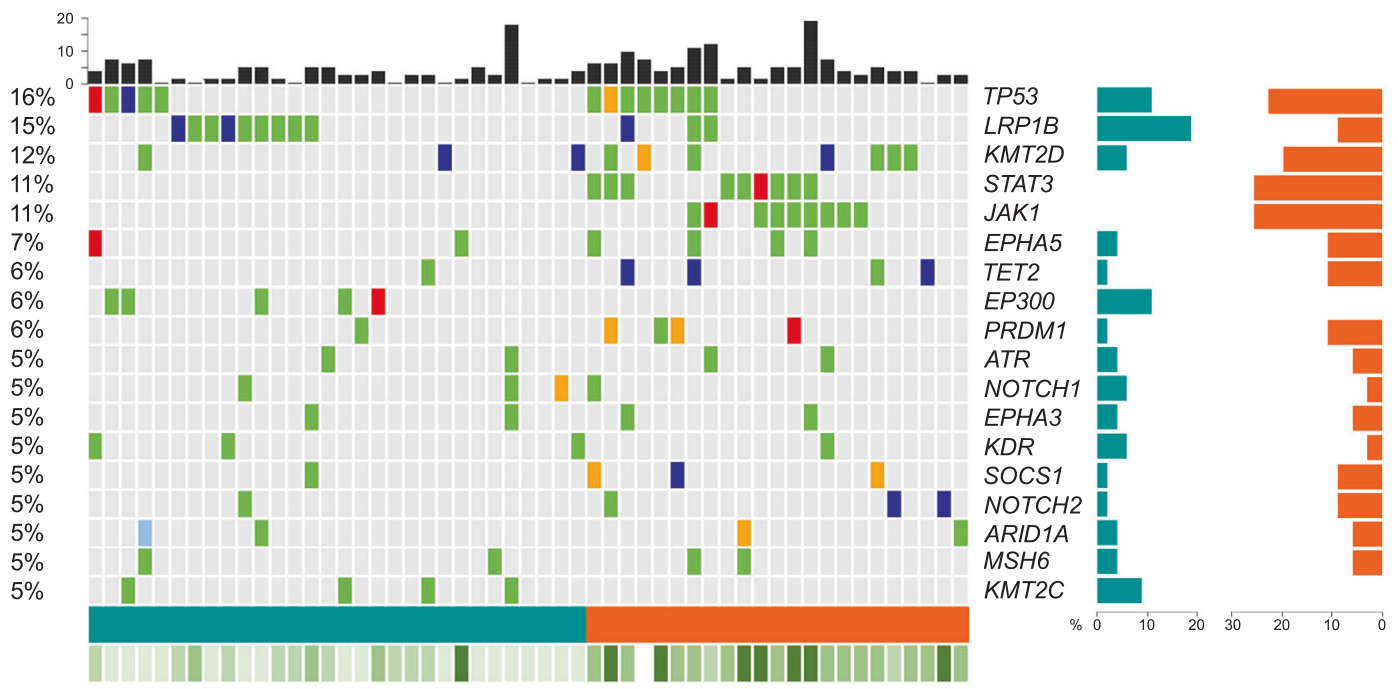

Entity
ALK+ patients
ALK- patients

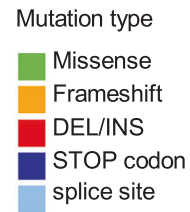

B
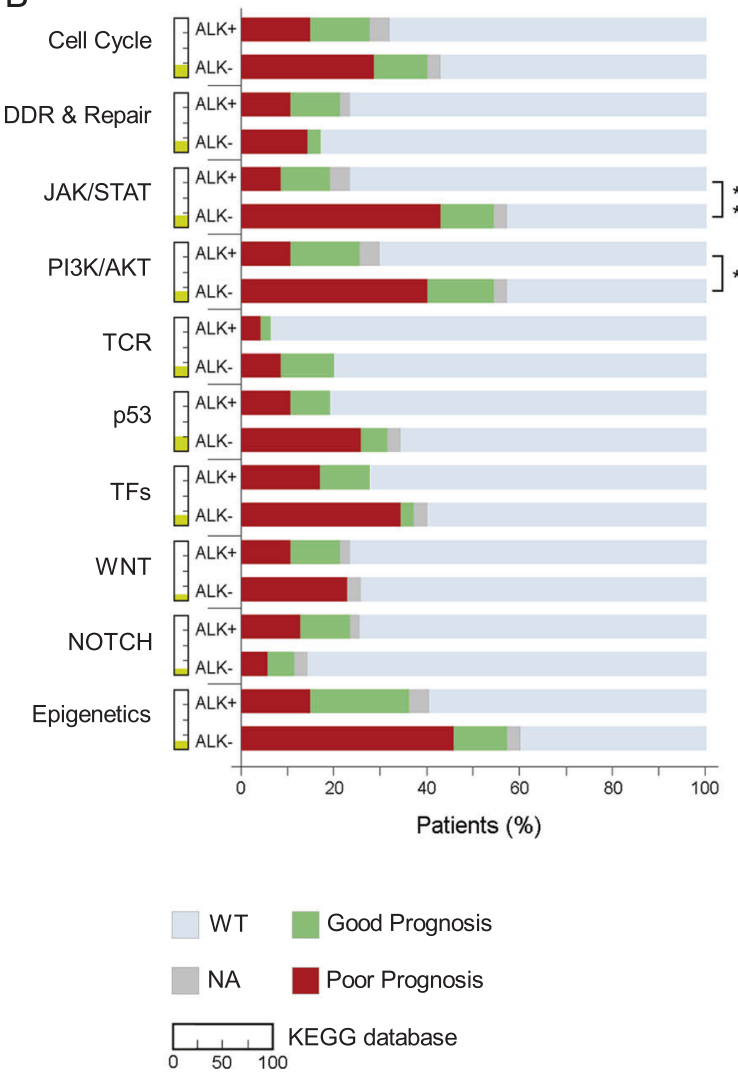

OS, more than $30 \%$ relapsed after first-line treatment. Among the 275 genes analyzed, we identified 148 (54\%) genes harboring at least one mutation throughout the entire

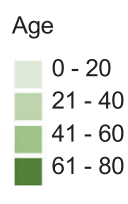

C

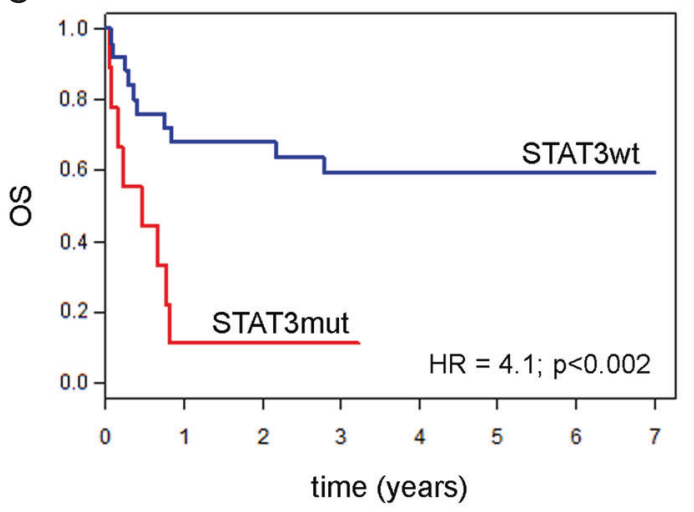

D
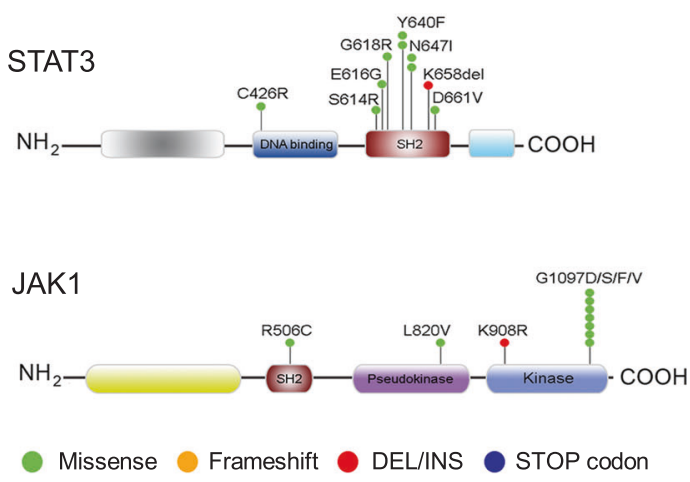

cohort; 132 genes among the patients and 43 among the cell lines, with 27 genes in common (Fig. S2, Table S3). Overall, 72 out of $82(88 \%)$ patients carried at least one 
Fig. 1 Mutational landscape in SALCL reveals prognostic biomarkers. a Oncoplot shows the genes mutated in at least 5\% of the entire cohort. The percentage is shown on the left axis. Each column represents a patient, ALK + in dark green and ALK - ALCL patients in dark orange. The black bars on the top represent the number of mutated genes in each patient. On the right axis, the frequency of mutated gene in ALK+ (dark green) and ALK - (dark orange) ALCL patients. The green bar on the bottom shows the age of each patient. Mutation types are represented in different colors as shown in the legend. b Percentage of patients harboring at least one mutated gene in ten biological pathways. The yellow colored portion next to each pathway indicates the percentage of genes present in our panel that belong to that specific pathway according to the KEGG database. For each pathway shown, the patients are divided according to prognosis; red: patients with poor prognosis; green: patients with good prognosis; gray: patients for whom clinical information is not available (NA); light blue patients wildtype (WT) that do not harbor mutated genes in that specific pathway. The patients are represented as percentage of the total. DDR and repair DNA damage response and repair pathway, TFs transcription factors. Fisher's exact test: $* p<0.05, * * p<0.01$ and $* * * p<$ 0.001. c 7-year OS of ALK - ALCL patients according to STAT3 status: STAT3 mutated (red) versus STAT3 wt (blue). $P$ values and hazard ratios (HR) shown were determined by the Cox proportional hazards model. d Schematic representation of STAT3 and JAK1 domains and the position of the variants.

mutation within the genes analyzed. We detected an average of 4.2 mutations per patient in ALK - ALCL and an average of 2.7 in ALK + ALCL. The most recurrently mutated gene in the entire cohort was TP53 found in $16 \%$ of sALCL patients $(11 \% \mathrm{ALK}+, 23 \% \mathrm{ALK}-$ and in all ALK + cell lines). Interestingly, for the ALK + group, mutated TP53 was more frequent in young patients $(p<0.04)$. LRP $1 B$ was prevalently mutated in ALK+ patients $(19 \%)$ and in three cell lines. STAT3 and JAK1 were mutated solely in ALKALCL, both with a prevalence of $26 \%$, and were the most mutated genes in this group (Fig. 1a). Recurrent mutations were detected in epigenetic modifier genes also recently reported to be frequently mutated in BIA-ALCL [14]. KMT2D and TET2 were found mutated in ALCL patients regardless of ALK status and EP30O and KMT2C only in ALK + patients. Pathway enrichment analysis showed a significant enrichment in mutated genes involved in JAK/ STAT $(p<0.003)$ and PI3K/AKT signaling pathways $(p<$ 0.02) for ALK - ALCL compared with ALK+ ALCL (Fig. 1b). We investigated possible correlations between the existence of mutations in the most mutated genes and the clinical characteristics of our cohort. Poor prognostic outcome was defined as patients meeting at least one of the following criteria: deceased, unresponsive to treatment and/ or disease relapse. The most recurrently mutated genes in the poor prognostic sub-cohort independent of ALK status were TP53 (27\%), STAT3 (24\%), EPHA5 (16\%), JAK1 (16\%), PRDM1 (13.5\%), LRPIB (11\%) and KMT2D (11\%). Considering only refractory/relapsed ALCL patients, mutations within TP53 (28\%) and EPHA5 (19\%) were the most common (Table $\mathrm{S} 4$ ). In relation to the prognosis, ALK
+ patients did not show any significant difference in the signaling pathways affected by mutations. On the contrary, the JAK/STAT $(p<0.005)$ and PI3K/AKT pathways $(p<$ 0.036) were enriched in ALK - ALCL patients with an inferior outcome (Fig. 1b). Pathogenetic variants of STAT3 were detected in $9 / 35(26 \%)$ of ALK - ALCL patients. Mutations were located mainly within the $\mathrm{SH} 2$ domain (S614R，E616G，Y640F， N647I， K658delinsNM and $\mathrm{D} 661 \mathrm{~V})$ and in one case within the DNA binding domain (C426R). Mutated JAK1 was detected in 9/35 (26\%) of ALK - ALCL patients and of those, 6/9 were at the hotspot codon 1097 (G1097D/F/N/S) (Fig. 1d). For four patients, $J A K 1$ was mutated together with STAT3, thereby emphasizing the importance of the JAK/STAT signaling axis. To evaluate the prognostic value of mutations in the JAK/ STAT pathway, we performed Cox regression analysis and showed that ALK - ALCL patients harboring STAT3 and/or $J A K 1$ mutation have a shorter OS (hazard ratio $[\mathrm{HR}]=2.8$; $95 \%$ confidence interval $[\mathrm{CI}], 1.1-7.1, p<0.03$ ) (Fig. S3A). Furthermore, the prognostic value of the most mutated genes in ALK - ALCL: STAT3 (9/35), JAK1 (9/35), TP53 (8/35) and KMT2D (7/35) were investigated. Cox regression analysis showed that patients with STAT3 mutations have a significantly shorter OS compared to those with wild-type STAT3 $\quad(\mathrm{HR}=4.1 ; \quad 95 \% \quad \mathrm{CI}, \quad 1.56-10.71, \quad p<0.002)$ (Fig. 1c). In addition, while $J A K 1$ and $K M T 2 D$ mutations did not significantly correlate with OS $(p<0.2$ and $p<0.3$, respectively), TP53 mutations clearly displayed the correlation $(p<0.01)$ (Fig. S3B-D). To further confirm that mutations in STAT3 are associated with shorter OS, we applied Akaike's informative criteria model to the four aforementioned genes. STAT3 mutations were found to be the best predictor of OS in ALK- ALCL (Table S5). Moreover, no significant differences were found between mutation status of these genes with age, gender, disease stage, eastern cooperative oncology group performance status or age-adjusted international prognostic index (AAIPI). As expected [9, 13, 15], expression of p-STAT3 (Y705) was detected at a high level in all ALK- ALCL patients harboring STAT3 mutations, although low/medium expression of p-STAT3 was also detected in STAT3 wildtype patient tumors (Fig. S4, Table S6). Mutations in the LRPIB gene were detected in $12 / 82$ (15\%) of sALCL patients $(19 \% \mathrm{ALK}+$ and $9 \% \mathrm{ALK}-)$ and three cell lines. Since $L R P 1 B$ was the most recurrently altered gene in ALK + ALCL, we assessed its possible association with outcome, but no differences were found between mutated and nonmutated patients.

To investigate somatic mutations with a possible role in disease relapse, we sequenced paired diagnostic and relapse samples available for four patients (1 ALK + and $3 \mathrm{ALK}-$ ) (Fig. 2a). Two different acquired mutations in EPHA5 were detected in each of the two relapse samples (patient tumors 
A
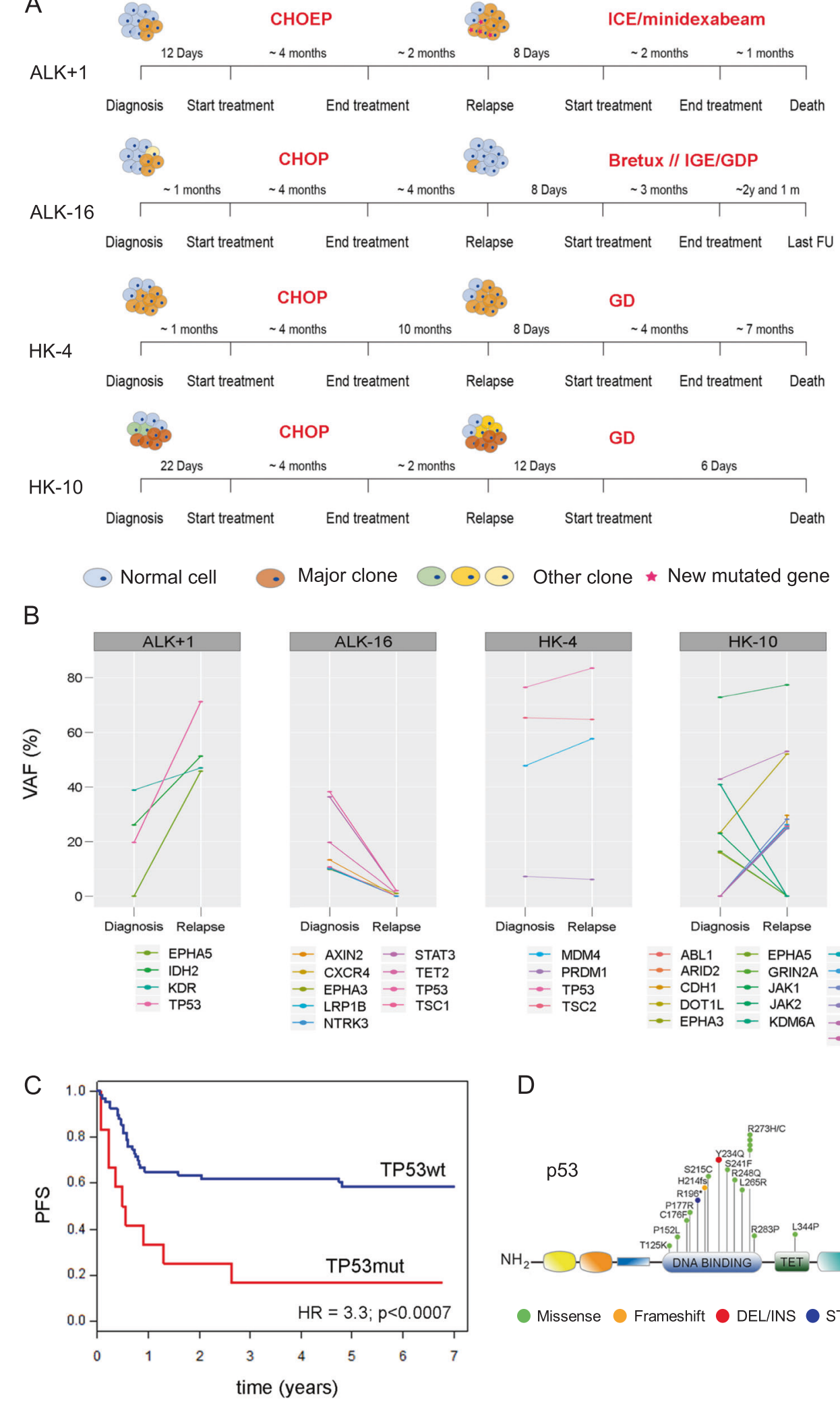

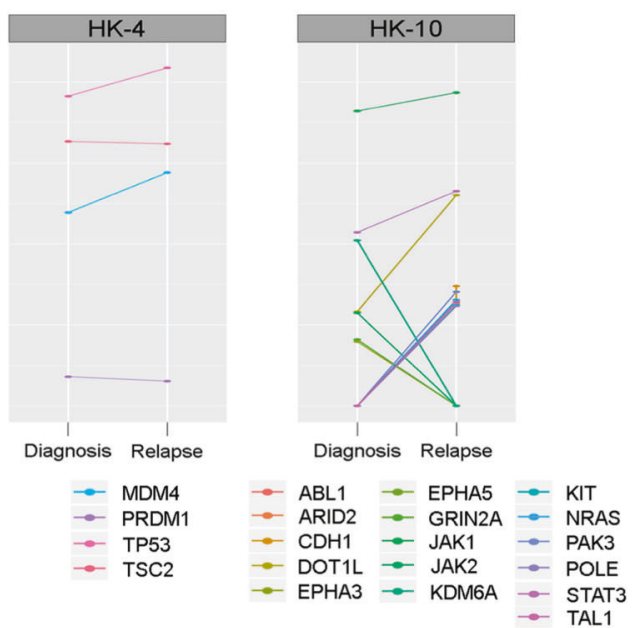

D

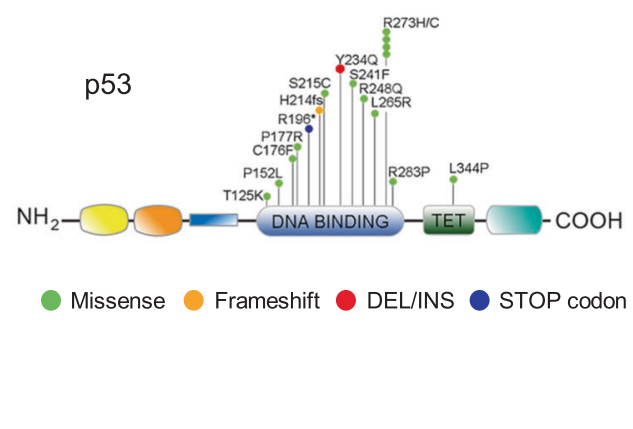

result of the emergence of a new malignant clone, harboring novel mutations in several other genes consistently with a similar variant allele frequency (Fig. 2b). Interestingly, glycine-valine change at residue 723 . In the latter patient (HK-10), identification of mutated EPHA5 appears to be the 
Fig. 2 Diagnosis versus relapse showed mutated TP53 to be associated with a shorter PFS in SALCL. a Schematic representation of four patients sequenced at diagnosis and at relapse highlighting their clinical path and treatment. Meaning of the color is described in the legend. CHOEP chemotherapy with cyclophosphamide, doxorubicin, etoposide, vincristine and prednisone, CHOP chemotherapy with cyclophosphamide, doxorubicin, vincristine and prednisone, ICE chemotherapy combination that includes ifosfamide, carboplatin and etoposide, bretux: brentuximab vedotin, GDP gemcitabine, dexamethasone, and cisplatin, GD gemcitabine and docetaxel, FU follow up. b Changes in mutational burden during tumor progression. The percentage of variant allele frequency (VAF\%) for each gene is plotted at diagnosis and at relapse. The names of genes involved are reported under each plot. $\mathbf{c} 7$-year PFS in systemic ALCL patients according to TP53 status; red: TP53 mutated (mut) patients; blue: TP53 wild-type (wt) patients. $P$ values and hazard ratios (HR) shown were determined by Cox proportional hazards. d Schematic representation of p53 domains and the variants detected.

EPHA5 was also found to be the second most mutated gene in relapsed/refractory patients in the entire cohort (Table S4B). Three out of four patients harbored mutated TP53 both at diagnosis and at relapse (Fig. 2b). As TP53 is the most recurrent gene mutated in our cohort and the most mutated gene in relapsed patients (Table S4B), we investigated its possible association with the treatment outcome for all ALCL patients regardless of ALK status. Nearly all mutations in TP53 were detected in the DNA binding domain except for L344P in the TET domain for one patient (Fig. 2d). sALCL patients harboring TP53 mutations have a shorter PFS compared to those with the wild-type gene $(\mathrm{HR}=3.3 ; 95 \%$ CI, 1.59-6.87, $p<0.0007)$ (Fig. 2c). These data, together with the diagnosis versus relapse analysis, suggest that TP53 mutations may confer resistance to chemotherapy. Moreover, mutations in TP53 were the most common genetic events on re-analysis of publicly available datasets (Table S7) $[9,11]$.

Losses at the genomic regions that encompass TP53 and PRDM1 genes have been shown to be the most common lesions in sALCL with a clinical implication [12]. PRDMI mutations were detected in five patients, with three of these co-occurring with TP53 mutations and all five patients being categorized within the poor prognostic sub-group. These data confirm the correlation between TP53 and PRDM1 gene mutations, thereby demonstrating either copy number loss or concomitant mutations are mechanisms which have the potential to alter p53 and PRDM1 pathways activity.

In summary, within one of the largest cohort of 82 sALCL patients, we provide robust information on the genetic spectrum of genes either solely mutated in ALKALCL (STAT3, JAK1) or across the whole spectrum of ALCL (TP53, LRP1B, EPHA5, KMT2D). In addition, we describe novel biomarkers for predicting treatment outcome reporting an association between mutated STAT3 and TP53 with an inferior outcome, in the former case in ALK- disease and in the latter case all sALCL independent of ALK status. Finally, this mutational landscape provides further candidate genes that deserve consideration for their possible role in the patient outcome, such as EPHA5, KMT2D, PRDM1 and SOCS1.

Acknowledgements This project has received funding from the European Union's Horizon 2020 Marie Skłodowska-Curie Innovative Training Networks (ITN-ETN) under grant agreement no. 675712. The project was also supported by Czech Science Foundation (GACR), GA19-15737S and junior project no. 19-23424Y, the MEYS CZ project CEITEC 2020 (LQ1601) and project MH CZ-DRO (FNBr, 65269705). We acknowledge the CF Genomics CEITEC MU supported by the NCMG research infrastructure (LM2018132 funded by MEYS CR) and Core Facility Bioinformatics of CEITEC MU for their support with scientific data presented here. We thank Vasileios Bikos and Stephen Paul Ducray for the support and advice. We acknowledge the different biobanks for collecting the samples and providing access to them: the CCLG Tissue Bank and contributing CCLG Centres, members of the ECMC Paediatric Network, the Czech National Lymphoma Registry (NiHiL, NCT 03199066), University Hospital Brno (grant no. NV18-03-00054) and University Hospital Hradec Kralove (Project BBMRI-CZ, no.: EF16 013/0001674).

Author contributions Conceptualization: CL; methodology: CL and BT; performed bioinformatics and statistical analysis: VB and LR; performed and interpreted IHC data: I-AM-M and FF; investigation: CL, AJ and SP; visualization: $\mathrm{CL}, \mathrm{LR}$ and $\mathrm{VB}$; writing — original draft: CL; writingreview and editing: SDT and SP; funding acquisition: OM, SDT, AJ and SP; collected data and samples: CL, DF, MM, NP, HL, SDT, H-CL, OM, KK, DB, GGS, LM, CG-P and AJ and supervision: SP.

\section{Compliance with ethical standards}

Conflict of interest The authors declare that they have no conflict of interest.

Publisher's note Springer Nature remains neutral with regard to jurisdictional claims in published maps and institutional affiliations.

Open Access This article is licensed under a Creative Commons Attribution 4.0 International License, which permits use, sharing, adaptation, distribution and reproduction in any medium or format, as long as you give appropriate credit to the original author(s) and the source, provide a link to the Creative Commons license, and indicate if changes were made. The images or other third party material in this article are included in the article's Creative Commons license, unless indicated otherwise in a credit line to the material. If material is not included in the article's Creative Commons license and your intended use is not permitted by statutory regulation or exceeds the permitted use, you will need to obtain permission directly from the copyright holder. To view a copy of this license, visit http://creativecommons. org/licenses/by/4.0/.

\section{References}

1. Savage KJ, Harris NL, Vose JM, Ullrich F, Jaffe ES, Connors JM, et al. ALK- anaplastic large-cell lymphoma is clinically and immunophenotypically different from both ALK + ALCL and peripheral Tcell lymphoma, not otherwise specified: report from the International Peripheral T-Cell Lymphoma Project. Blood. 2008;111:5496-504. http://www.ncbi.nlm.nih.gov/pubmed/18385450. 
2. Janikova A, Chloupkova R, Campr V, Klener P, Hamouzova J, Belada D, et al. First-line therapy for T cell lymphomas: a retrospective population-based analysis of $906 \mathrm{~T}$ cell lymphoma patients. Ann Hematol. 2019. http://link.springer.com/10.1007/ s00277-019-03694-y.

3. Hapgood G, Savage KJ. The biology and management of systemic anaplastic large cell lymphoma. Blood. 2015;2:17-25. https:// pubmed.ncbi.nlm.nih.gov/25869285/.

4. Brugières L, Deley MC Le, Pacquement H, Meguerian-Bedoyan Z, Terrier-Lacombe MJ, Robert A, et al. CD30+ anaplastic largecell lymphoma in children: analysis of 82 patients enrolled in two consecutive studies of the French Society of Pediatric Oncology. Blood. 1998;92:3591-8.

5. Mussolin L, Damm-Welk C, Pillon M, Zimmermann M, Franceschetto G, Pulford K, et al. Use of minimal disseminated disease and immunity to NPM-ALK antigen to stratify ALK-positive ALCL patients with different prognosis. Leukemia. 2013;27:416-22. http://www.ncbi.nlm.nih.gov/pubmed/22907048.

6. Morel A, Brière J, Lamant L, Loschi M, Haioun C, Delarue R, et al. Long-term outcomes of adults with first-relapsed/refractory systemic anaplastic large-cell lymphoma in the pre-brentuximab vedotin era: a LYSA/SFGM-TC study. Eur $\mathrm{J}$ Cancer. 2017;83:146-53.

7. Wrobel G, Mauguen A, Rosolen A, Reiter A, Williams D, Horibe $\mathrm{K}$, et al. Safety assessment of intensive induction therapy in childhood anaplastic large cell lymphoma: Report of the ALCL99 randomised trial. Pediatr Blood Cancer. 2011;56:1071-7. http://www.ncbi.nlm.nih.gov/pubmed/21280197.

8. Prokoph N, Larose H, Lim MS, Burke GAA, Turner SD. Treatment options for paediatric anaplastic large cell lymphoma (ALCL): current standard and beyond. Cancers. 2018;10:1-18.
9. Crescenzo R, Abate F, Lasorsa E, Tabbo’ F, Gaudiano M, Chiesa $\mathrm{N}$, et al. Convergent mutations and kinase fusions lead to oncogenic STAT3 activation in anaplastic large cell lymphoma. Cancer Cell. 2015;27:516-32. https://linkinghub.elsevier.com/retrieve/pii/ S153561081500094X.

10. Larose H, Prokoph N, Matthews JD, Schlederer M, Högler S, Alsulami AF, et al. Whole exome sequencing reveals NOTCH1 mutations in anaplastic large cell lymphoma and points to Notch both as a key pathway and a potential therapeutic target. Haematologica. 2020. http://www.haematologica.org/lookup/doi/10. 3324/haematol.2019.238766.

11. Song TL, Nairismägi ML, Laurensia Y, Lim JQ, Tan J, Li ZM, et al. Oncogenic activation of the STAT3 pathway drives PD-L1 expression in natural killer/T-cell lymphoma. Blood. 2018;132:1146-58.

12. Boi M, Rinaldi A, Kwee I, Bonetti P, Todaro M, Tabbò F, et al. PRDM1/BLIMP1 is commonly inactivated in anaplastic large Tcell lymphoma. Blood. 2013;122:2683-93.

13. Andersson EI, Brück O, Braun T, Mannisto S, Saikko L, Lagström S, et al. STAT3 mutation is associated with STAT3 activation in CD30+ ALK - ALCL. Cancers. 2020;12:702. https://www.mdpi.com/2072-6694/12/3/702.

14. Laurent C, Nicolae A, Laurent C, Le Bras F, Haioun C, Fataccioli $\mathrm{V}$, et al. Gene alterations in epigenetic modifiers and JAK-STAT signaling are frequent in breast implant-associated ALCL. Blood. 2020;135:360-70.

15. Shen J, Li S, Medeiros LJ, Lin P, Wang SA, Tang G, et al. PD-L1 expression is associated with ALK positivity and STAT3 activation, but not outcome in patients with systemic anaplastic large cell lymphoma. Mod Pathol. 2020;33:324-33. https://www.na ture.com/articles/s41379-019-0336-3. 